\title{
Research on improved algorithm of Hoff circle detection
}

\author{
Xing Zhang ${ }^{1, a}$,Wei Lin $^{2, b}$ \\ School of Guangdong University of Technology, Guangzhou 510000, China. \\ a799351412@qq.com
}

Keywords: Recognition accuracy, detect circles,Formation about Gradient.

\begin{abstract}
For the original random Hoff transform (RHT) in a small color image, the detection accuracy of the shortage of the circle, and if uncontrolled on the radius of the circle, not only the poor accuracy, but also a huge amount of computation.In order to improve the accuracy of finding circle, compared to the fitting, it need to provide additional parameters to control, parameter requirements more stringent, and poor overall stability. I proposed a modified RHT to find out the number of candidate circles after the vote, and then draw the circles, And comparison with the actual Contour,sorting by the actual total number of pixels overlap, then the highest score of the circle is the target circle.This method can solve the problem of low recognition accuracy. The following experiments we can see the error is improved, accuracy improvement has been very good. in improving the same time, it did not increase the amount of calculation. the algorithm has a high recognition accuracy, wide range, and strong anti-interference.
\end{abstract}

\section{Introduction}

Detection is one of the basic research issues in pattern recognition and image processing[1].Most of the current algorithms are based on the Hoff gradient algorithm.Jia Ping and Xu Ning proposed a gray histogram based on local features to reduce the dimension of feature vectors[2].Applicable in different light, the angle is relatively fast, the accuracy is still not good enough.And vision is more and more used in industrial robots[3,4].However, there are some problems such as low accuracy of calculation, simple work pieces, demanding background and complex calibration process[5,6].

In this paper, starting from the stochastic gradient Hoff transform method, mainly according to the gradient of the round and a final vote of candidates, by these candidate round pixel in the actual contour pixels of the proportion of the total to judge whether they are the best circle detection.

\section{Principle of Hoff transform circle}

Circle transform Hoff law about transform similar line Hoff law. Hoff line transformation method is determined by two parameters of R, theta, and line detection method is similar, on the Hoff circle detection method, determined by the three parameters and than the test line to more complicated, parameters $\mathrm{X}$ and $\mathrm{Y}$ parameter mapping for the coordinates of the center, $\mathrm{R}$ is said required for detection of the three parameters i.e. radius, are as follows (1) the three parameters $\mathrm{C} 1$, C2 and C3 decision.

$$
\left(x-c_{1}\right)^{2}+\left(y-c_{2}\right)^{2}=c_{3}^{2}
$$

Corresponding to any point, in the coordinate system are corresponding to the $\mathrm{C} 1, \mathrm{C} 2, \mathrm{C} 3$ composed of three-dimensional space of the space surface. And on the C1, C2, C3 plane of a point, the corresponding to the $\mathrm{Y} x$ plane it is a circle.Corresponding to the $\mathrm{Y}, \mathrm{X}$ plane of three points ( $\mathrm{x} 0$, $\mathrm{Y} 0),(\mathrm{x} 1, \mathrm{Y} 1),(\mathrm{X} 2, \mathrm{Y} 2)$, in $\mathrm{C} 1, \mathrm{C} 2, \mathrm{C} 3$ three dimensional space is corresponding to the three spatial surfaces (2), (3), (4) .at this point, C1, C2, C3 is equivalent to a constant.

$$
\begin{aligned}
& \left(x_{0}-c_{1}\right)^{2}+\left(y_{0}-c_{2}\right)^{2}=c_{3}{ }^{2} \\
& \left(x_{1}-c_{1}\right)^{2}+\left(y_{1}-c_{2}\right)^{2}=c_{3}{ }^{2}
\end{aligned}
$$




$$
\left(x_{2}-C_{1}\right)^{2}+\left(y_{2}-c_{2}\right)^{2}=c_{3}^{2}
$$

Solving these three equations, we can get $\mathrm{C} 1, \mathrm{C} 2, \mathrm{C} 3$ value. This shows that $(\mathrm{x} 0, \mathrm{Y} 0),(\mathrm{x} 1, \mathrm{Y} 1)$, (X2, Y2) of the three points in the $\mathrm{C} 1, \mathrm{C} 2$, C3 determined on a circle, the center coordinates and radius and the above three points after $\mathrm{C} 1, \mathrm{C} 2, \mathrm{C} 3$ is determined by the space of three space curved surface on. Further, in the XY plane, three points in the same circle, then their corresponding surfaces intersect at one point (C1, C2, C3).

So if we know the number of a point on the boundary enough, and these points and the corresponding spatial surface intersect at one point. These points constitute the boundary, is close to a round.

\section{Hoff gradient algorithm improvement}

\subsection{Shortcomings of previous algorithms}

According to the analysis of the classical Hoff transform and its improved algorithm, the shortcomings in the reaction of 3 dots in the following:

(1) Non target edge points and noise for detection, sensitivity although not so high, but on detection efficiency and accuracy influence still role can not be ignored;

(2) In the case of the target and the background color is small, Hoff gradient voting may not be optimal round round out. As shown in Fig. 2. Point A,B,C votes more than the actual center;

(3) However, the increase of non target points will have a greater impact on the hit rate of random sampling, which leads to large deviation of target recognition. Is not ideal, as shown in Fig. 1 below.

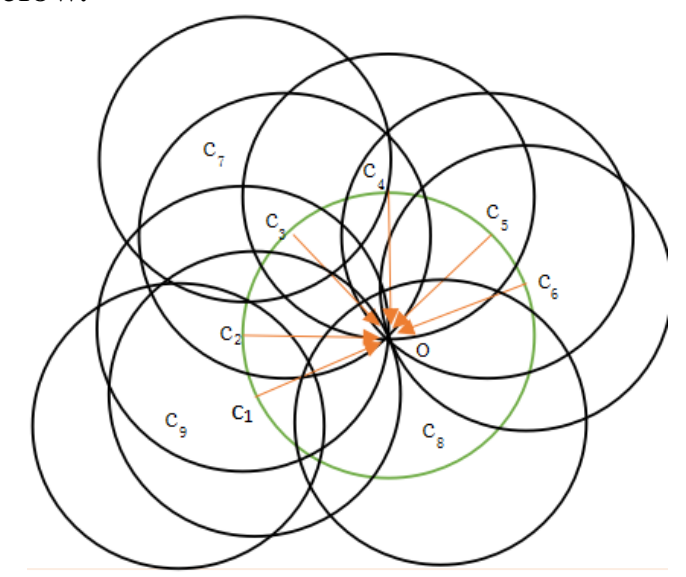

Fig. 1 Ideally voting center

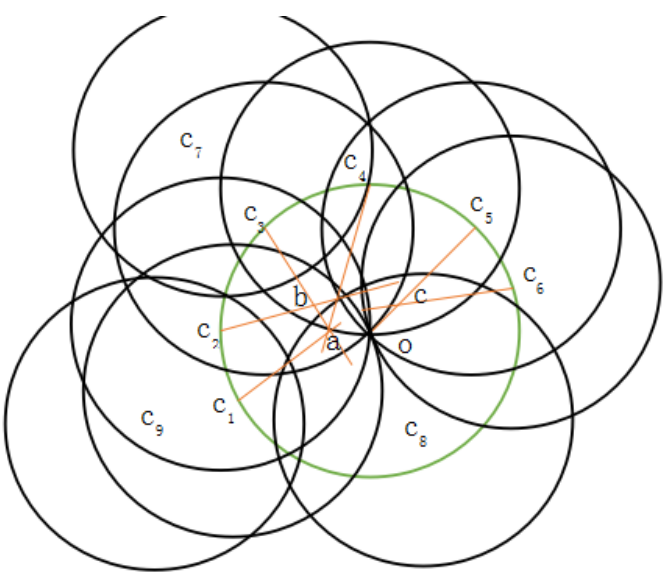

Fig. 2 Actual situation round voting

\subsection{Algorithm improvement idea}

Idea of the algorithm is put forward an improved RHT to find a group of almost round and then draw the circle, and the actual contour than, sorted by total number of actual overlap pixel, then the highest score of the round target circle. Thus, the problem of low recognition accuracy is solved. Algorithm the main task from a single vote out round (possibly not optimal circle), as long as the pixel points reach a certain number of real circle as the confirmation, change to vote for the highest number of round (several or more than ten) pixel match the highest degree of round, namely real circle. At this time, the optimal round may not the center of the circle the circle that the highest vote.

\section{Experiment}

\subsection{Experimental Process}

Experiment of the software platform: the preparation of software Studio Visual 2008 MFC, the operating system 64 bit window7.

The default of the best circle under the Hoff gradient is shown in Fig. 4 (by default in the order of the cumulative value of the poll results). 


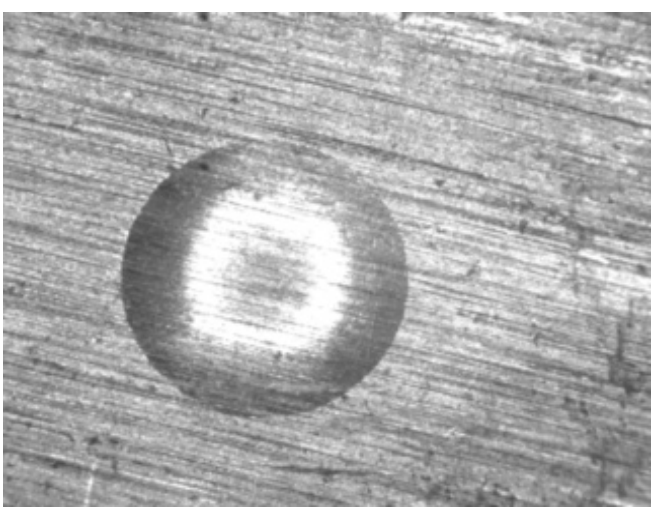

Fig. 3 Original picture

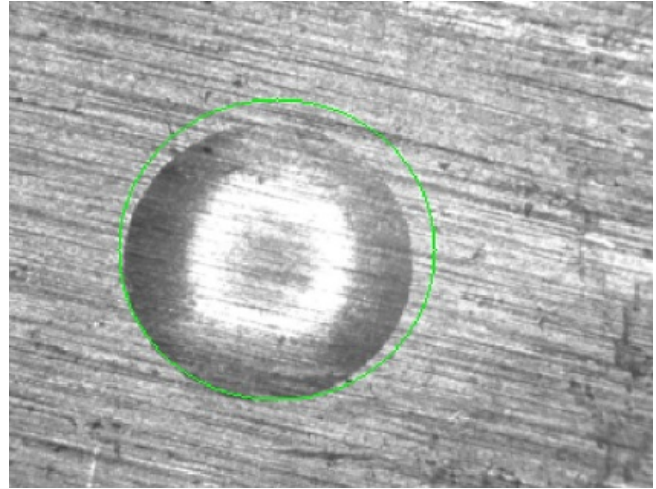

Fig. 4 the best round By default

But to study the optimization algorithm itself, contour gradient mapping out the central vote originally precision is low (Fig. 4), find out round there will be many is wrong, but if a sufficient number of contour points, find the right round must also exist, just according to the number of votes method to evaluate possible sorting by comparison, but after all, is there; here the a clever optimization, change the evaluation method, scheduling optimization, the result is very close to the Fig. 6

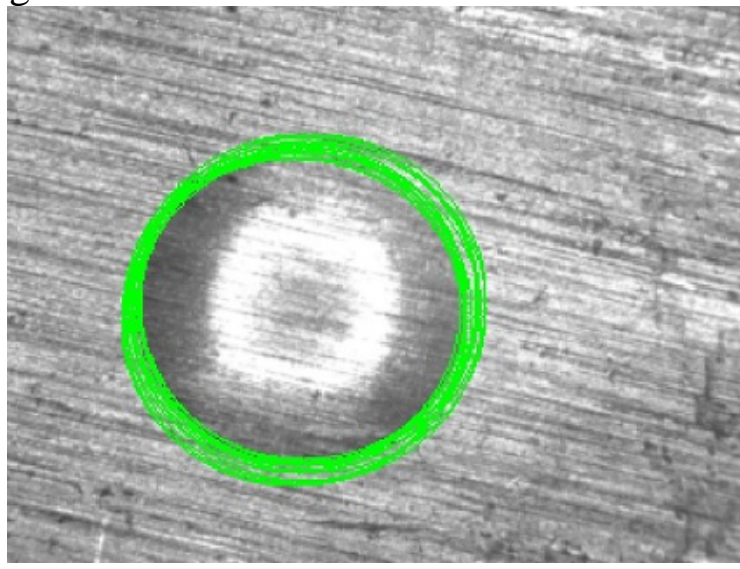

Fig. 5 Round of voting, the top

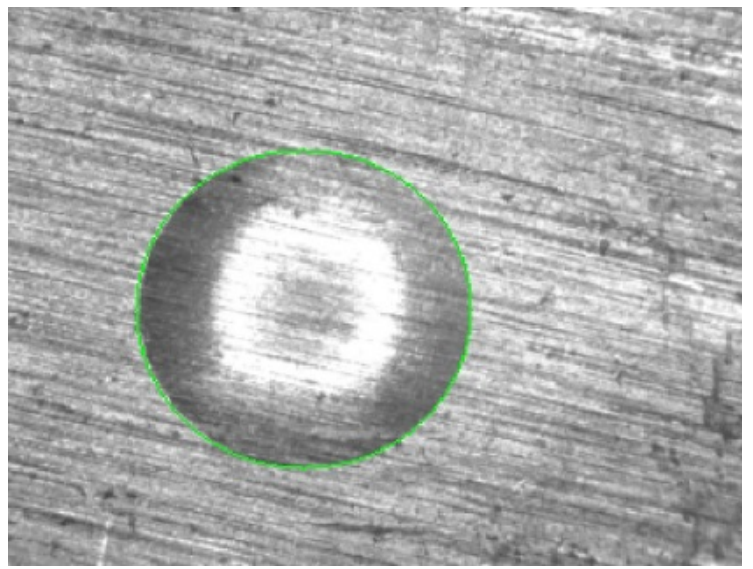

Fig. 6 Round optimized

\section{Summary}

Seriously consider the randomized Hoff transform circle and improved gradient circle transformation, and in careful consideration of the other circle detection method to improve, this paper presents a new gradient of RHT circle detection based on improved algorithm, by finding out by a group vote almost round (Fig. 5) and then draw the circle, and the actual contour than, sorted by total number of actual overlap pixel, then the highest score of the round target circle. Thus, the problem of low accuracy of Hoff gradient is solved well. Experiments show that the improved algorithm has a good accuracy, and the error can achieve the desired effect. This algorithm has the advantages of strong anti-interference ability, high recognition accuracy, suitable for all kinds of environments, and widely used.

\section{References}

[1]. Davies E R. Computer and Machine Vision: Theory, Algorithms, Practicalities. B Jain Publishers. Vol. 18(2012) No. 5,p. 78-82.

[2]. Jia P, Ning X U, Zhang Y. Automatic target recognition based on local feature extraction. Optics \& Precision Engineering. Vol. 21(2013) No. 7,p. 1898-1905.

[3]. Yan Z G, Ming L I, Ke-Fei X U, et al. High-speed Robot Auto-sorting System Based on Machine Vision. Packaging \& Food Machinery. Vol. 12(2014) No. 1,p. 28-31. 
[4]. Wang H X, Xiang-Ru L I, Xue-Cheng S U. Applied positioning method for hand-eye vision system. Computer Engineering \& Applications. Vol. 18(2007) No. 24,p. 235-238.

[5]. Zhang J, Yang X F, Zhao R L. Eyes Detection Based on Integral Projection and Hough Transform. Journal of Electron Devices. Vol. 28(2005) No. 27,p. 43-44.

[6]. LIN Zhi-qiang.Real-time Object Recognition System Combining Multiple Feather.Journal of Chinese Computer System.Vol.3(2015) No. 6,p. 1310-1315. 